\title{
An IoT-Focused Intrusion Detection System Approach Based on Preprocessing Characterization for Cybersecurity Datasets
}

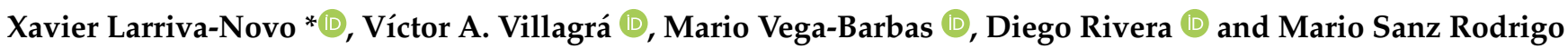 \\ ETSI Telecomunicación, Universidad Politécnica de Madrid (UPM), Avda, Complutense 30, 28040 Madrid, Spain; \\ victor.villagra@upm.es (V.A.V.); mario.vega@upm.es (M.V.-B.); diego.rivera@upm.es (D.R.); \\ mario.sanz@upm.es (M.S.R.) \\ * Correspondence: xavier.larriva.novo@upm.es
}

Citation: Larriva-Novo, X.; Villagrá, V.A.; Vega-Barbas, M.; Rivera, D.; Sanz Rodrigo, M. An IoT-Focused Intrusion Detection System Approach Based on Preprocessing Characterization for Cybersecurity Datasets. Sensors 2021, 21, 656. https://doi.org/10.3390/ s21020656

Received: 17 December 2020

Accepted: 16 January 2021

Published: 19 January 2021

Publisher's Note: MDPI stays neutral with regard to jurisdictional claims in published maps and institutional affiliations.

Copyright: (c) 2021 by the authors. Licensee MDPI, Basel, Switzerland. This article is an open access article distributed under the terms and conditions of the Creative Commons Attribution (CC BY) license (https:// creativecommons.org/licenses/by/ $4.0 /)$.
Abstract: Security in IoT networks is currently mandatory, due to the high amount of data that has to be handled. These systems are vulnerable to several cybersecurity attacks, which are increasing in number and sophistication. Due to this reason, new intrusion detection techniques have to be developed, being as accurate as possible for these scenarios. Intrusion detection systems based on machine learning algorithms have already shown a high performance in terms of accuracy. This research proposes the study and evaluation of several preprocessing techniques based on traffic categorization for a machine learning neural network algorithm. This research uses for its evaluation two benchmark datasets, namely UGR16 and the UNSW-NB15, and one of the most used datasets, KDD99. The preprocessing techniques were evaluated in accordance with scalar and normalization functions. All of these preprocessing models were applied through different sets of characteristics based on a categorization composed by four groups of features: basic connection features, content characteristics, statistical characteristics and finally, a group which is composed by traffic-based features and connection direction-based traffic characteristics. The objective of this research is to evaluate this categorization by using various data preprocessing techniques to obtain the most accurate model. Our proposal shows that, by applying the categorization of network traffic and several preprocessing techniques, the accuracy can be enhanced by up to $45 \%$. The preprocessing of a specific group of characteristics allows for greater accuracy, allowing the machine learning algorithm to correctly classify these parameters related to possible attacks.

Keywords: Internet of Things; machine learning; intrusion detection system; preprocessing techniques; traffic categorization

\section{Introduction}

Cyberspace plays a fundamental role in the society and economy, as the Internet has changed the means of communication for people or organizations. Furthermore, different devices, applications, and services which are linked to cyberspace are included inside the term Internet of Things (IoT). So, information communication technology (ICT) has been intensively applied for the deployment of various types of sensors actuators, for machineto-machine (M2M) communications infrastructures [1], with the principal objective of processing the huge amount of data that is provided for these services or applications.

According to the process of gathering information, transmission, and processing from IoT systems, IoT has an entity-based architecture that is divided in three main layers. These layers are usually named: terminal perception layer, network transport layer, and application service layer [2]. The terminal perception layer is composed by the source of IoT data collection. The units involved in this layer include physical entities representing real devices units (sensors devices, identification devices, tracking and positioning devices). Furthermore, the network transport layer transmits the information gathered by the perception layer to the application service layer. Finally, the application service layer process 
the data transmitted from the network transport layer to various industries or entities, providing services for different users through different fields, such as smart grids, smart homes, and smart cities [2].

The network transport layer can suffer several security threats, such as distributed denial of service (DDoS) attacks, sending traffic and consuming network and computing resources [3]. Additionally, the application layer from IoT systems is vulnerable to several types of cybersecurity attacks, such as Worms, Trojan, DoS, or Spyware. These types of attacks are becoming more sophisticated, increasing its number day to day [4]. Additionally the application of different standardized communications combined with the limited computer power and the high number of connected devices, can make the traditional security countermeasures not efficient in IoT systems [5]. This research is focused on the detection of possible attacks between the transport network layer and the application layer as presented in Figure 1, which is detailed through this research.

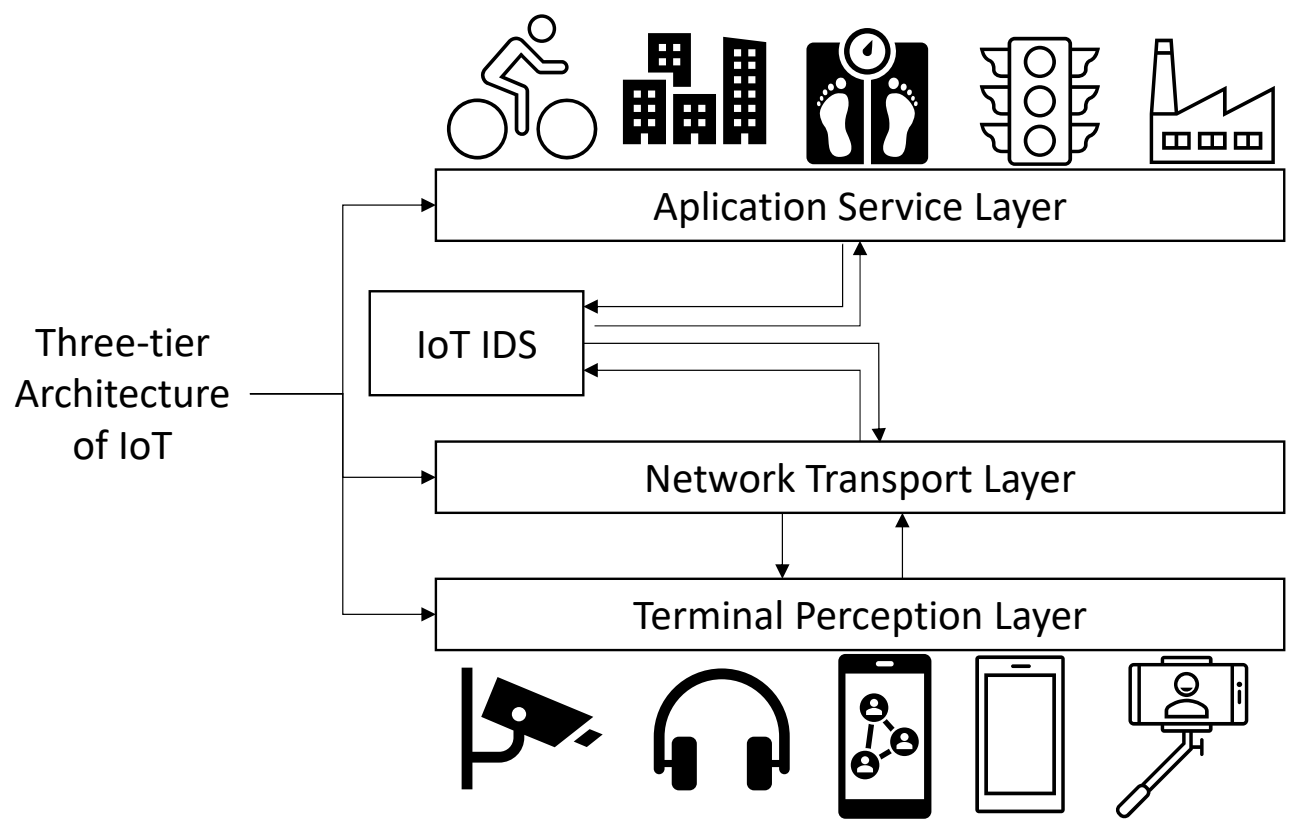

Figure 1. Three-tier architecture of IoT and IoT IDS approach.

For this reason, developing security solutions for IoT is indispensable, with the objective to prevent and mitigate possible cybersecurity issues.

To detect IoT attacks on the network transport layer, networks intrusion detection systems (NIDS) have been deployed as a second line of defense after Firewalls, antivirus, and access control systems [6] for connected smart things. A NIDS is a software with functionalities focused on monitoring ICTs from fraudulent uses, unauthorized access or any other cyberattack. There exist different types of NIDS, including those based on signature detection and those aimed at detecting anomalies. NIDS oriented to anomaly detection are able to perform an examination of the network, verifying its behavior and activity, then being able to detect and catalog possible deviations of patterns that represent the malicious behavior of possible cyberattacks. Although such systems represent a robust solution for attack detection, they must deal with a major challenge that deteriorate their accuracy: the detection of false positives due to the similarities between legitimate and anomalous observations.

Thus, Machine Learning techniques, specifically deep learning (DL) algorithms, are being proposed as an effective solution for dealing with this problem $[7,8]$. One of the most important requirements of NIDS based on DL techniques is the preprocessing phase, which can affect the accuracy of an algorithm in a significant way [9]. This data preprocessing consists of transforming the input data by using different techniques such as One-Hot encoding [10], z-score [11], and standardization type min-max [12]. However, IoT ecosystems 
are delocalized, distributed and composed by a large number of devices with computing performance limited in resources. IoT is also limited by network bandwidth capacity. This implies that NIDS based on anomalies must be efficient and accurate. In this way, this article aims to identify the preprocessing level configuration that yields the best accuracy of the underlying model.

In addition, and taking into account that IoT systems try to reduce the computational cost as much as possible, strengthening the learning model and avoiding the possible overfitting to increase the efficiency of the underlying learning models, this research work proposes the use of the categorization defined in [13]. This categorization is composed by four groups of features which include basic connection features, content characteristics, statistical characteristics, and finally, a group which is composed by traffic-based features and connection direction-based traffic characteristics. The objective of this research is to evaluate this categorization by using various data preprocessing techniques based on transforming categorical values into numerical values and by applying standardization and normalization.

Finally, up-to-date benchmark datasets for IoT IDSs are currently almost non-existent [6,8], although some datasets have been generated for this environments, e.g., the IoTID20 [14]. As a consequence, for this research we have opted to use three of the most widely accepted and adopted benchmark datasets, such as KDD99 [15] and NSL-KDD [16], which is an improved version of the KDD99. Additionally, we use UNSW-NB15 [17], and UGR16 [18], a novel dataset which has been applied previously in the study of NIDS based on anomalies [9].

This paper introduces the background and related work to this research work during Section 2. Additionally, the paper introduces the proposal of a new way of data preprocessing based on traffic characterization for IDSs in Section 2. Furthermore, in Sections 3-6, we explain the problem statement and the proposed methodology, defining the methodology ML and preprocessing model presented in this paper. These sections also include the architecture proposed for applying the multilayer perceptron (MLP) and the obtained results after applying our proposal to the UNSW-NB15, UGR16, and KDD99 datasets. Finally, Section 5 includes the discussion of the main conclusions and future lines for this research.

\section{Background and Related Work}

\subsection{Data Threatment}

Data preprocessing applied to NIDS based on ML algorithms is divided into three main categories: data reduction, treatment of missing data, and data scaling.

Data reduction can be divided into feature selection and case selection. Feature selection is the process of selecting a subset of features that provide a similar impact in the results, rather than selecting the entire set of features of the selected dataset. Its main goal is to increase the accuracy of a ML algorithm and reduce the cost for the fitting and validation in terms of computer resources [19]. Case selection is similar to feature selection, being their main difference that case selection intends to identify and remove the redundant data from the dataset. This method allows to reduce the size of the dataset from its original dimensions, reducing the time required for the algorithm to be fitted and validated [20].

Treatment of missing data is also divided in data elimination and data imputation. The first one is composed by removal by lists and by pairs, while data imputation is divided into: mean imputation, hot-deck imputation, cold imputation and regression [21].

Data scaling is defined by the transformation of the data using diverse methods, i.e., standardization and normalization. This type of processing allows to transform the data from an established scaling function. Consequently, the values of a class of a dataset expresses the same degree of influence for the ML algorithm [22]. Taking this idea, some research works have introduced other aspects of preprocessing techniques based on four main components: feature selection, feature reduction, clustering, and hybrid approaches [23]. Nevertheless, these preprocessing techniques were only approached through the use of the KDD99 dataset. 


\subsection{Preprocessing Related Works and Network Iintrusion Detection Systems Data Preprocessing}

Several studies have been carried out in the area of data preprocessing, with the aim of optimizing the data used by different ML algorithms and ultimately to enhance the accuracy with less computing performance.

In this sense, Ref. [24] introduced different anomaly detection algorithms which were applied to the NSL-KDD dataset to evaluate different preprocessing techniques. The research applied standardization and normalization to the dataset obtaining an overall accuracy of $99 \%$. The research work presented in [25] evaluated the impact of different attribute normalization schemes on a combination of different features from the dataset NSL-KDD. The authors provided an evaluation of three different algorithms using six different characteristics where a K-nearest neighborhood obtained the best accuracy with $98.9 \%$, followed by a multilayer perceptron model with $96.5 \%$ and naïve Bayes with $93.3 \%$. The study remarked the importance of the attribute selection and normalization to increase the accuracy of the models. Authors in [26] proposed an NIDS based on anomaly detection, where categorical values were mapped into numerical values, while the non-categorical values were normalized in ranges between zero and one. Applying this procedure, the authors highlighted an improvement in the accuracy of the model proposed, achieving a maximum accuracy of $99.5 \%$ in denial-of-service attacks. The study conducted in [27] presented an evaluation of various configurations of data preprocessing. The authors proposed a standardization of type z-score of 12 attributes and 34 attributes normalization obtaining a maximum accuracy of $98 \%$ for the standardization model and $99 \%$ for the normalization model. The research proposed the use of a back propagation neural network: the concluding results introduce the standardization and selection of hyperparameters as the principal characteristics for enhancing the accuracy. Additionally, the mentioned research used the dataset KDD99.

As observed, different studies on the optimization of preprocessing data have been carried out, traditionally, by using the KDD99 or NSL-KDD dataset. In [28], authors presented a study that determined that the most commonly used datasets for the different analysis of NIDS based on anomalies detected by ML techniques are the KDD99 with $63.8 \%$ of popularity followed by the NSL-KDD with $11.6 \%$. In addition, this study proposed the application of an ML-based NIDS model for the detection of anomalies in IoT systems. In this sense, this research introduces the analysis of characteristics and preprocessing models based on normalization and scaling for two new benchmark datasets, the USW-NB15 and the UGR16. The research carried out and presented in this paper tries to identify the most suitable characteristics for developing ML-based NIDS models, reducing the processing time, and improving the accuracy for IoT environments.

\section{The Proposed Approach}

The proposed work presented in this research is designed to evaluate the set of characteristics proposed in [13]. The architecture proposed for the IDS consists in the introduction of individual preprocessing techniques based on a content characterization. The system is presented in Figure 2. As can be seen in the figure, it is composed by some phases for the evaluation in order to obtain the best accuracy. These stages are described in the following sections.

\subsection{Datasets under Study}

In relation to the datasets created for IoT networks, many studies use the KDD'99 dataset. This dataset has become a reference for various investigations related to NIDS for IoT networks [5]. However, in cybersecurity, this dataset is currently considered obsolete, due to the age of attacks it presents $[9,13]$. Several studies have compared different cybersecurity datasets, each one of these datasets being created through different methods, in the field of IDS. Also, nowadays, these datasets have been considered as benchmark datasets for the evaluation of IDS, and also applied to IoT IDS $[4,9,16-18,24,29,30]$. Therefore, an updated dataset with current attacks, UGR16 and real collected traffic with up-to-date 
attacks, is proposed as the basis for the analysis in this research. Additionally, to extend the analysis comparison, it is proposed to use two benchmark datasets, i.e., UNSW-NB15 and NSL-KDD.

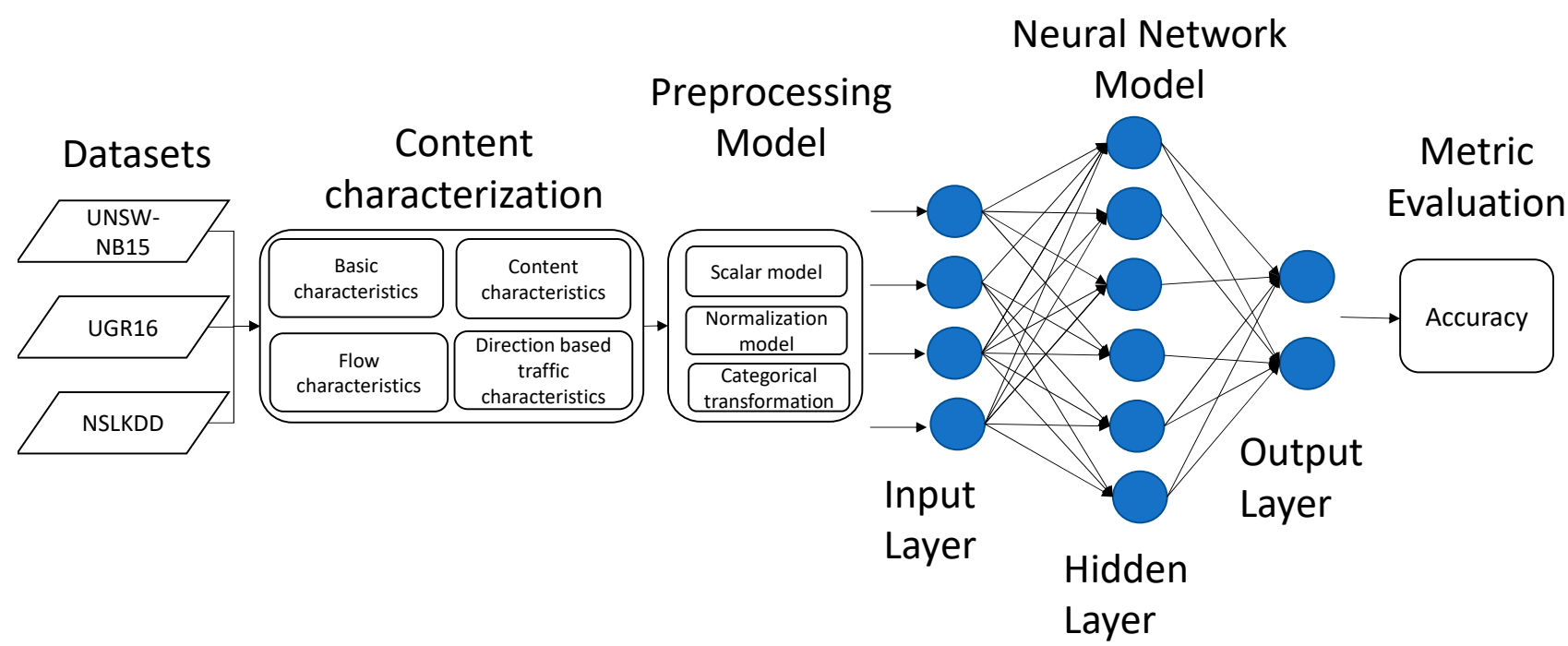

Figure 2. Categorical data preprocessing results in terms of accuracy scaled between 0 to 1 .

\subsubsection{Dataset UGR16}

The UGR16 [18] dataset is a more realistic attempt made at capturing NetFlow traces covering more than four months of network traffic from an internet service provider (ISP). An important advantage of this dataset is the normal traffic, that was adequately captured from different sensors located in the ISP networks. This dataset takes around 19.900 million of unidirectional flows offering a big scope for experimentation. Additionally this dataset is clean from synthetically generated attacks $[18,31]$. The UGR16 has 13 characteristics: timestamp of the end of a flow (time), flow duration (duration), source IP address (sip), destination IP (dip), source port (source port), destination port (destination port), protocol (protocol), flags (flags), forwarding status (forward_status), type of service (type_service), packets exchanged in the stream (pack_exchanged), their corresponding number of bytes (bytes), and the attack type (attack_tag).

There are multiple options for obtaining portions of the UGR' 16 dataset. In this case, the week of 2 August, test version, was taken as a sample. This was selected for the present study, due to the fact that the test versions have synthetic traffic which allows applying more varied data. The original version of the data set is $81 \mathrm{~GB}$. For this research, a 1.4 GB portion was selected, making it proportional to the original dataset in terms of attack and normal traffic proportions. Table 1 shows the relationship between normal traffic and attacks from the UGR16 dataset.

Table 1. Proportions of attacks and no attack for UGR16 dataset.

\begin{tabular}{cc}
\hline Category & Percentage \\
\hline Attack & $12.82 \%$ \\
No Attack & $87.18 \%$ \\
\hline
\end{tabular}

All the features mentioned above were considered for the proposed model, except for the time feature. That feature was eliminated since the evaluation of the NIDS as a model based on time series is not within the scope of this research. Therefore, the research was carried out with 12 basic characteristics. 


\subsubsection{Dataset UNSW-NB15}

The UNSW-NB15 [17] dataset has 49 features classified into 5 categories: flow characteristics, basic characteristics, content characteristics, time characteristics, and generated additional characteristics. Table 2 shows the relationship between normal traffic and attacks from the UNSW-NB15 dataset

Table 2. Proportions of attacks and no attack for UNSW-NB15 dataset.

\begin{tabular}{cc}
\hline Category & Percentage \\
\hline Attack & $44.94 \%$ \\
No Attack & $55.06 \%$ \\
\hline
\end{tabular}

- The basic characteristics, unlike in NSL-KDD, include the attributes that represent the protocol connections, most of them similar to the basic characteristics in NSL-KDD.

- The flow characteristics include the identifying attributes between the hosts.

- Content characteristics involve TCP/IP attributes and some http connections.

- The time characteristics contain all the attributes related to time i.e., arrival time between packets.

- Additional characteristics generated divided into two groups, i.e., general purpose to protect the service of protocols and connection characteristics.

\subsubsection{Dataset NSL-KDD}

The NSL-KDD dataset is an improved version of the KDD99. It not only solves the redundant records problems of the KDD99, but also makes the number of traces appropriate in the training and testing dataset [32]. This clean version prevents the machine learning algorithm from being biased during the training data phase [33]. Finally, thanks to this dataset and the high number of studies from the last decade, we can still make a comparison for suitable algorithms related to the last decade. The NSL-KDD dataset has 42 characteristics, classified into 3 categories: basic characteristics, content characteristics, and traffic characteristics. Table 3 shows the relationship between normal traffic and attacks from the NSL-KDD dataset.

Table 3. Proportions of attacks and no attack for NSL-KDD dataset.

\begin{tabular}{cc}
\hline Category & Percentage \\
\hline Attack & $46.54 \%$ \\
No Attack & $53.56 \%$ \\
\hline
\end{tabular}

- The basic characteristics include all the attributes that can be extracted from an individual TCP/IP connection.

- The content characteristics consist of some specific characteristics necessary to detect attacks that show suspicious behavior in the data portion, for example, number of failed login attempts.

- Flow characteristics include the characteristics calculated with respect to a window interval.

\subsection{Data Preprocessing}

Data preprocessing consists in transforming the data values of a certain dataset, aiming to optimize the information acquisition and process. Normally, there is a very large contrast between the maximum and minimum values of the dataset, so normalizing the data minimizes the complexity of the algorithm for its corresponding processing. According to [27], the normalization of the data allows an adequate benefit for the classification of algorithms related to neural networks. In this case, if the back-propagation technique is used in neural networks, the normalization of the input values will speed up the training phase, turning it into a more efficient neural network. 


\subsubsection{Normalization Function}

The main normalization function is based on data scaling, which consists of the minmax algorithm, which is capable of converting the current range of data typically in the interval $[-1,1]$ and $[0,1]$. The normalization formula is presented in Equation (1).

$$
p=\frac{\left(\left(x-x_{\min }\right)(\max -\min )\right.}{\left(x_{\max }-x_{\min }\right)+\min }
$$

where $(\min , \max )$ is the specified range of input variable, $\left(x_{\min }, x_{\max }\right)$ the initial range of values of input variables, and $p$ is the converted input value.

\subsubsection{Standardization Function}

The standardization function, or z-score, is able to normalize the features of the dataset. It has the properties to normalize the features values of a dataset, normalizing a standard distribution. These values are represented in Equation (2), where $\mu$ is the mean (the average value of a feature over all the values of that feature in the dataset) and $\partial$ is the standard deviation of the mean.

$$
x^{\prime(j)}=\frac{x^{(j)}-\mu^{(j)}}{\partial^{(j)}}
$$

\subsection{Deep Learning Algorithm under Study}

The multi-layer perceptron neural network (MLPNN) consists in a linear classification algorithm capable of ordering the input data into categories. MLPNN are feed-forward neural networks that consists of a large number of neurons classified into input units (input layer), output units (output layer) and hidden units (hidden layer). The weights assigned to the connections are estimated using a back-propagation algorithm. The values of the weights define the performance of the neural network.

In this article we only focus on supervised learning, due to the specific problem stated in it. The proposed model is limited to analyzing the sequence of the attacks because the objective of the study is not focused temporal time series. Thus, the main objective is to identify the optimization of characteristics proposed in [13] for diverse datasets at the level of preprocessing. For this, the MLPNN architecture proposed in this research was designed according to the criteria established in the aforementioned study, defining an MLP model and its associated best hyperparameters.

The configuration for the neural network taken as the basis for the comparison in this study consists of a 4-layer network. The input layer with a neuron density corresponding to the input data of each dataset, the hidden layers with a density equal to the rule that obtained the best precision in the research mentioned below, and an output layer with an equal neuronal density according to the attack classification are proposed in this research. The last layer was defined by a density equal to one, since a binary class classification between attack and no-attack will be obtained at the output. For the model proposed in this study, the initialization of the weights was done applying Glorot normal initializer [34] with no seed. Furthermore, the computational experiments were averaged with the function Earlystoping [35] setting the loss with a min_delta of $10^{-3}$ in order avoid the overtraining of the algorithm. The patience was set to 5 (number of epochs with no improvements after the training is stopped). This value was settled after several test in order to improve the computation costs of the training. Finally, the best weights were obtained setting the variable restore_best_weithgs to True which is capable to restore to the model from the epoch with the best value of the monitored loss

\subsection{Evaluation Metrics}

Accuracy (AC) is considered one of the most important performance indicators. As one of the most used metrics in several works as was presented during Section 2. This metric determines the number of records in a class predicted correctly. The value of true positive (TP) is equivalent to the correctly predicted values, corresponding to a class. The false 
positive (FP) value is the number of predictions that are not equivalent to the corresponding class. The true negative (TN) is the result of those values that are presented, corresponding to the number of records that are identified as normal. The false negative (FN) is the incorrectly predicted result for a corresponding class. Equation (3) shows how the AC and relationship of these parameters is calculated. Table 4 presents the corresponding confusion matrix.

$$
\mathrm{AC}=\frac{\mathrm{TP}+\mathrm{TN}}{\mathrm{TP}+\mathrm{TN}+\mathrm{FP}+\mathrm{FN}}
$$

Table 4. Confusion matrix for the actual class vs. the predicted class.

\begin{tabular}{lll}
\hline & \multicolumn{3}{c}{ Predicted Class } \\
\hline \multirow{2}{*}{ Actual Class } & TP & FN \\
\cline { 2 - 3 } & FP & TN \\
\hline
\end{tabular}

\section{Methodology and Experimentation}

\subsection{Entire Set of Characteristics Evaluation}

Each dataset considered for performing the experimentation of this research is composed by different characteristics presented during Section 3. The main proposal at this point is to determine the best data preprocessing function by proposing the transformation of categorical variables into numerical data and the standardization and normalization functions for the underlying model.

The neural network algorithm proposed for this case study does not allow the use of text-type input variables, so these variables are transformed into binary vectors by the onehot encoding [36] method and the attacks were transformed into binary vectors. Table 5 presents the proposal of non-numerical data that were transformed using the different methods mentioned above.

Table 5. Categorical data transformations for the datasets understudy.

\begin{tabular}{|c|c|c|}
\hline Dataset & One-Hot Encoding & Binary Vector \\
\hline UGR16 & protocol, flag & attack_tag \\
\hline NSL-KDD & $\begin{array}{l}\text { protol_type, service, flag, land, } \\
\text { num_failed_login, is_host_login, is_guest_login }\end{array}$ & attack_tag \\
\hline UNSW-NB15 & dur, proto, service & attack_tag \\
\hline
\end{tabular}

All the datasets were divided into training and testing datasets where the $75 \%$ of the entire dataset was considered for the training and the $25 \%$ for the testing. These datasets were applied with the function of train_test_split [37] with defined random state [38] variable for all the datasets.

As a first point of analysis, the study is carried out using the proposed datasets submitted to the same architecture of the neural network, without pre-processing the data. Only the categorical variables shown in Table 6 were transformed. This was carried out to obtain a base precision measure, which would allow us to determine the increase in precision based on the various types of preprocessing techniques applied.

The results obtained from the training and validation of the proposed algorithm show that the NSL-KDD dataset offers a better accuracy, with $95.5 \%$, compared to $87.68 \%$ of the UGR16 and $55.80 \%$ of the UNSW-NB15. These values are represented in Figure 3. It should be noted that the architecture of the neural network is the same for all datasets as described in Section 3.3. 
Table 6. Variations in terms of accuracy according to preprocessing techniques for the NSL-KDD dataset.

\begin{tabular}{cccc}
\hline Basic Characteristics & $\begin{array}{c}\text { Content } \\
\text { Characteristics }\end{array}$ & Flow Characteristics & Accuracy \\
\hline z-score & min_max_0 & Z-score & 0.97923414 \\
min_max_0 & min_max_0 & z-score & 0.978567346 \\
z-score & min_max_0 & min_max_0 & 0.97694799 \\
min_max_0 & z-score & z-score & 0.974693592 \\
z-score & z-score & min_max_0 & 0.971550137 \\
min_max_0 & z-score & min_max_0 & 0.970851591 \\
min_max_0 & - & z-score & 0.970121293 \\
min_max_0 & z-score & z-score & 0.969867276 \\
z-score & z-score & min_max_0 & 0.964628183 \\
min_max_0 & z-score & - & 0.946434241 \\
\hline
\end{tabular}

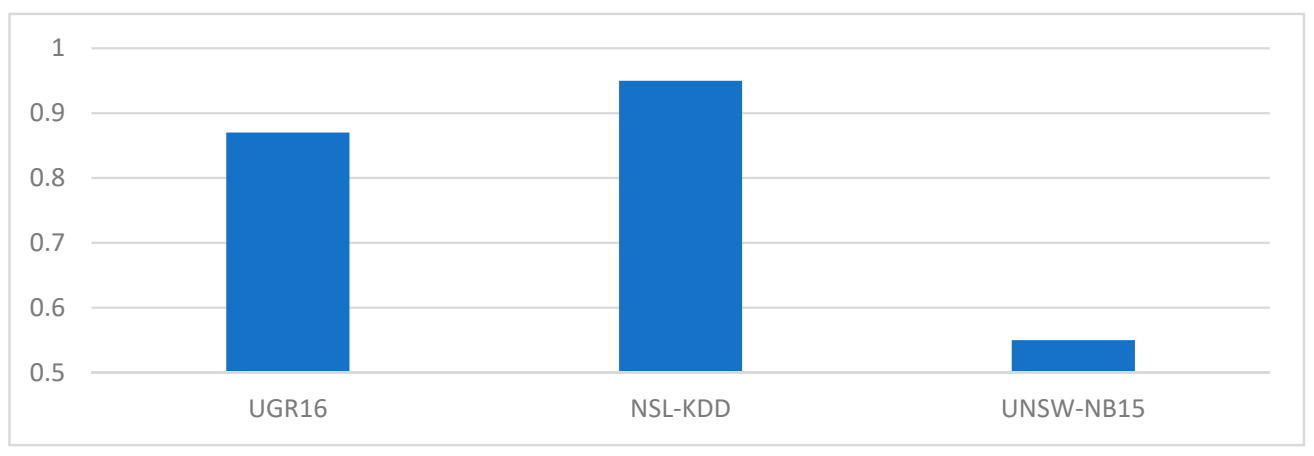

Figure 3. Categorical data preprocessing results in terms of accuracy scaled [0-1].

In order to carry out the evaluation of the proposed preprocessing methods, the first approach consists in evaluating the total set of features for each individual dataset, testing them with the same preprocessing technique. The total set of features evaluated with the standardization function corresponds with the label z_score_all, following with the normalization functions: minmax_0_all for the configuration with a minimum of 0 and a maximum of 1 and minmax_1_all for the configuration with a minimum of -1 and a maximum of 1.

The evaluation presented an increase in terms of accuracy for each individual dataset. In case of UGR16, an accuracy of $99.3 \%$ was obtained by applying z_score_all; while the accuracy was increased to $99.88 \%$ when applying the minmax_0_all configuration; and correspondingly for the minmax_1_all configuration the accuracy was reduced to $99.18 \%$.

The same methodology was applied for the NSL-KDD dataset with the configurations: z_score_all, minmax_0_all, and minmax_1_all, where values of $97.89 \%, 96.25 \%$, and $96.48 \%$ of accuracy were obtained, respectively.

Finally, the UNSW-NB15 dataset was evaluated with the same configuration mentioned above, that is, z_score_all, minmax_0_all, and minmax_1_all, and producing values of $98.3 \%, 98 \%$, and $98.2 \%$ in terms of accuracy respectively. All of these results are summarized in Figure 4.

The experimentation exposed that the preprocessing techniques could enhance up to $45 \%$ the accuracy in respect to the no preprocessing techniques, such as the case for the UNSW-NB15 dataset. Furthermore, it can be seen that z_score_all configuration technique for all groups of characteristics provides better results for all the datasets under study. 


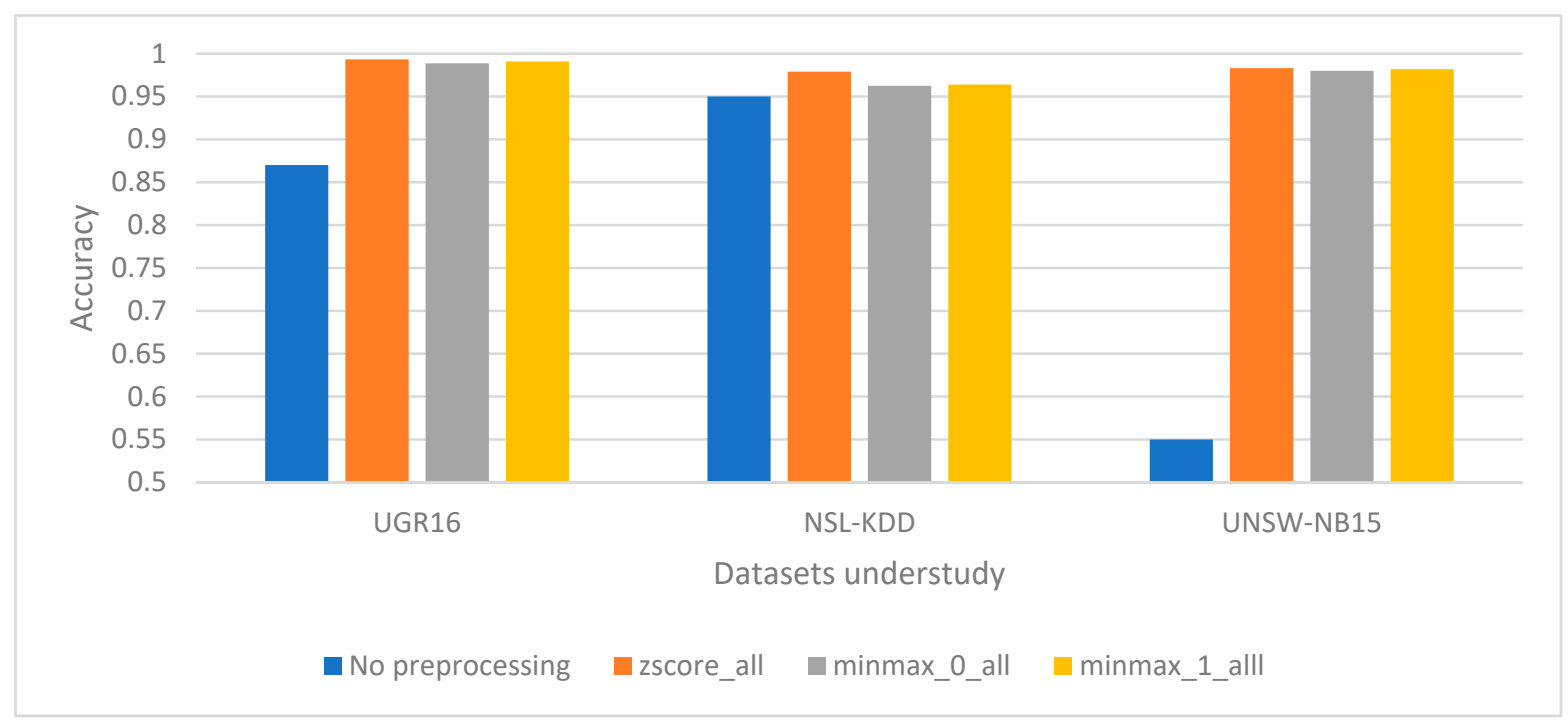

Figure 4. Standardization and normalization functions applied for the entire group of characteristics versus no processing functions for the UGR16, NSL-KDD and UNSW-NB15 datasets accuracy scaled between 0 to 1.

\subsection{Individual Set of Characteristics Evaluation}

As mentioned before, the objective of this research work proposes the use of the categorization defined in [13] which is defined by four groups characteristics. This categorization was applied to the entire set of features of each proposed dataset under study mentioned previously. Additionally, three preprocessing techniques were applied individually for each individual set of characteristics, in order to compare the results. Specifically, the standardization (z-score), normalization ( $\min -\mathrm{max})$, and no preprocessing (-) technique were applied.

In the case of the UGR16 dataset, since it only has 12 basic characteristics, three evaluations were carried out, which were those mentioned in Section 4.1 and exposed in Figure 2. In the case of the NSL-KDD and UNSW-NB15 datasets, a complete comparison of preprocessing techniques was possible because they present diverse groups of characteristics, as it was shown in Section 3. Thus, results obtained for NSL-KDD and UNSW-NB15 are presented and analyzed.

Table 6 shows the 10 main accuracy variations with the most favorable results obtained, arranged in descending order applying the variations of the data preprocessing techniques for each of the groups of characteristics of the NSL-KDD dataset. These data were characterized according to the proposal in [13]. However, this dataset does not contain direction-based traffic characteristics, so the evaluation of preprocessing techniques was done without this group of characteristics.

Table 7 shows the accuracy variations of the applied preprocessing techniques for the various sets of characteristics presented in the UNSW-NB15 dataset, presenting the 10 best variations ordered in descending order according to the validation score.

Table 8 shows the best configurations obtained with the highest precision for each dataset (UNSW-NB15, NSL-KDD and UGR16) by groups of characteristics. This table shows that the standardization to the group of basic characteristics and statistical traffic characteristics allows increasing the accuracy of the algorithm. Unfortunately, a direct comparison between the content characteristics cannot be obtained, since for the UNSW-NB15 dataset and for the NSL-KDD dataset their preprocessing gives a non-significant precision variation. Since the UGR16 dataset contains only basic characteristics, it allows determining a substantial increase in precision with the use of the standardization algorithm. 
Table 7. Variations according to preprocessing techniques for the UNSW-NB15 dataset.

\begin{tabular}{|c|c|c|c|c|}
\hline $\begin{array}{c}\text { Basic } \\
\text { Characteristics. }\end{array}$ & $\begin{array}{c}\text { Flow } \\
\text { Characteristics }\end{array}$ & $\begin{array}{c}\text { Content } \\
\text { Characteristics }\end{array}$ & $\begin{array}{c}\text { Direction-Based } \\
\text { Traffic } \\
\text { Characteristics }\end{array}$ & Accuracy \\
\hline z-score & z-score & z-score & min_max_0 & 0.992 \\
\hline z-score & min_max_0 & min_max_0 & min_max_0 & 0.9908 \\
\hline z-score & - & - & - & 0.8638 \\
\hline \multirow[t]{2}{*}{ z-score } & z-score & z-score & - & 0.8618 \\
\hline & & z-score & - & 0.8349 \\
\hline - & z-score & z-score & z-score & 0.7869 \\
\hline - & z-score & z-score & - & 0.7163 \\
\hline - & - & - & z-score & 0.6581 \\
\hline - & z-score & - & - & 0.5775 \\
\hline
\end{tabular}

Table 8. Variations according to preprocessing techniques for the proposed datasets.

\begin{tabular}{|c|c|c|c|c|c|c|}
\hline Configuration & Dataset & $\begin{array}{c}\text { Basic } \\
\text { Characteristics }\end{array}$ & $\begin{array}{c}\text { Content } \\
\text { Characteristics }\end{array}$ & $\begin{array}{c}\text { Flow } \\
\text { Characteristics }\end{array}$ & $\begin{array}{c}\text { Direction- } \\
\text { Based Traffic } \\
\text { Characteristics }\end{array}$ & Accuracy \\
\hline N01 & NSL-KDD & Z-score & min_max_0 & z-score & & 0.997 \\
\hline N02 & NSL-KDD & min_max_0 & min_max_0 & z-score & & 0.978 \\
\hline N03 & NSL-KDD & z-score & z-score & z-score & & 0.978 \\
\hline N04 & NSL-KDD & & & & & 0.95 \\
\hline N05 & UGR16 & Z-score & & & & 0.993 \\
\hline N06 & UGR16 & & & & & 0.87 \\
\hline N07 & UNSW-NB15 & z-score & z-score & z-score & min_max_0 & 0.992 \\
\hline N08 & UNSW-NB15 & min_max_0 & min_max_0 & z-score & min_max_0 & 0.990 \\
\hline N09 & UNSW-NB15 & z-score & z-score & z-score & z-score & 0.983 \\
\hline N10 & UNSW-NB15 & & & & & 0.55 \\
\hline
\end{tabular}

Additionally, we have evaluated the most accurate models for each dataset i.e., N01, N05 and N07, presented in Table 8. These models were evaluated with the confusion matrix in term of percentage as is presented in Table 9. The percentage was considered related to the total number of flows from each dataset previously evaluated. Finally, these models were also evaluated with other metrics such as precision and recall as presented in Table 10.

Table 9. Confusion Matrix in terms of percentage for the most accurate models.

\begin{tabular}{|c|c|c|c|c|c|c|c|}
\hline \multirow{3}{*}{$\begin{array}{l}\text { True } \\
\text { Label }\end{array}$} & & \multicolumn{2}{|c|}{ Model N01 } & \multicolumn{2}{|c|}{ Model N05 } & \multicolumn{2}{|c|}{ Model N07 } \\
\hline & Attack & $47.94 \%$ & $0.03 \%$ & $11.55 \%$ & $0.49 \%$ & $43.31 \%$ & $0.39 \%$ \\
\hline & No Attack & $0.36 \%$ & $51.67 \%$ & $0.14 \%$ & $87.82 \%$ & $1.18 \%$ & $55.12 \%$ \\
\hline & & Attack & No Attack & Attack & No Attack & Attack & No Attack \\
\hline & & \multicolumn{6}{|c|}{ Predicted Label } \\
\hline
\end{tabular}

Table 10. Recall and Precision for the most accurate models presented during this research.

\begin{tabular}{cccc}
\hline & Model N01 & Model N05 & Model N07 \\
\hline Precision & 0.992 & 0.988 & 0.973 \\
Recall & 0.999 & 0.959 & 0.990 \\
\hline
\end{tabular}

\section{Discussion}

The preprocessing of the basic characteristics allows greater accuracy, because a mean distribution of the values is generated, allowing the ML algorithm to correctly identify these parameters related to attacks. 
Research works presented in Section 2 were analyzed looking for the existence of preprocessing techniques such as scalar, normalization and categorical transformation. The comparison presented in Table 11 exposes the best results obtained in terms of accuracy between the works mentioned before and our proposed models for each one of the analyzed datasets in through this research.

Table 11. Variations according to preprocessing techniques for the proposed datasets.

\begin{tabular}{|c|c|c|c|c|c|c|}
\hline \multirow{2}{*}{$\begin{array}{c}\text { Research and } \\
\text { Configuration Proposed }\end{array}$} & \multicolumn{3}{|c|}{ Preprocessing Technique } & \multirow{2}{*}{$\begin{array}{l}\text { ML Model } \\
\text { Applied }\end{array}$} & \multirow[t]{2}{*}{ Dataset } & \multirow[t]{2}{*}{ Accuracy } \\
\hline & Scalar & Normalization & Categorical & & & \\
\hline N01-Model proposed & Yes & Yes & Yes & $\mathrm{NN}$ & NSL-KDD & 0.997 \\
\hline N05-Model proposed & Yes & Yes & Yes & $\mathrm{NN}$ & UNSW-NB15 & 0.992 \\
\hline N07-Model proposed & Yes & Yes & Yes & $\mathrm{NN}$ & UGR16 & 0.993 \\
\hline Paulauskas et al. & No & Yes & No & $\mathrm{NN}$ & NSL-KDD & 0.99 \\
\hline Salih et al. & No & Yes & No & $\mathrm{NN}$ & NSL-KDD & 0.989 \\
\hline Lokeswari et al. & Yes & No & Yes & NN & KDD99 & 0.99 \\
\hline Chiba et al. & Yes & Yes & No & NN & KDD99 & 0.98 \\
\hline
\end{tabular}

The results obtained determine that our proposal gets better results with the preprocessing techniques commonly used and the assumed group of characteristics. Most of the recent studies have been developing models for benchmarks datasets such as KDD99 or NSL-KDD. In this way, the principal difference is that our proposal enhances the detection rate in terms of accuracy for individual groups of characteristics through different datasets. Furthermore, this characterization may improve a comparison in terms of precision between different benchmark and non-benchmark datasets taking into account the similarity of the proposed NetFlow characterization of characteristics.

Thus, the NIDS based on ML presented in this paper offer several advantages over other models. First, it is able to easily detect an attack, correctly differentiating it from normal traffic. Second, thanks to the use of benchmark and up-to-date datasets, the proposed model is capable of facing sophisticated attacks. These advantages, together with the classification proposed in [13] have allowed the proposed ML model to improve the accuracy, which is essential for its deployment in real environments.

After performing the experiments described above with the proposed neural network model, it is possible to obtain an acceptable precision greater than $99 \%$ in each of the datasets used. This shows the benefit of data preprocessing based on the categorization that we propose, demonstrating that a data standardization based on the scaling of variables using the z-score algorithm allows increasing the precision of the ML algorithm for the model.

Concretely, a high precision was obtained with the proposed model and the various types of preprocessing models. Table 7 shows the diverse configurations applied and the enhance up to $44 \%$ in the accuracy of the algorithm when preprocessing the information properly, this specifically in the case of the UNSW-NB15 dataset. The actual variation in precision depends on the ML model proposed and its hyperparameters, so this study focuses on the application of a single model to the various datasets, which is a way of remark the contribution of the preprocessing techniques proposed by group of characteristics.

\section{Conclusions and Future Lines}

This research work introduces new comparisons between the use of various datasets such as the case of UNSW-NB15 and UGR16, allowing to extend the various studies in the application of ML algorithms for the case of anomaly-based NIDS. In all the datasets previously studied, the transformation of categorical values to numerical values is essential, as this ensures that the data can be correctly coupled to the ML algorithm. Furthermore, the study shows that data preprocessing task is very important for the ML algorithm to obtain 
greater precision in terms of the classification of anomalies, as can be seen by comparing Figures 3 and 4.

The most relevant conclusion that this study provides is the importance of preprocessing characteristics, such as basic characteristics and statistical traffic characteristics using z-score standardization techniques, which allows increasing precision since it allows using the mean deviation of the variables. As future actions, we will extend this research and we intend to apply the proposed model and preprocessing functions by individual group of characteristics into a real environment, with real data collected from IoT systems, such as the platform of Smart City services proposed in [1], to demonstrate the efficiency of our implementation. This implementation could be done with technologies such as Structured Streaming from Spark [39], which is able to replicate the neural network and preprocessing models proposed for streaming data applications in real time. In this way, the IDS proposed would be able to detect real attacks with features related to each one of the datasets evaluated during this research. Finally, as a future work, we will continue this research, using a complete version of the UGR16 and big data technologies, taking some considerations from related researches such as the amount of redundant records of the datasets used in this article.

Author Contributions: Conceptualization, V.A.V. and X.L.-N.; methodology, X.L.-N. and V.A.V.; software, X.L.-N. and M.S.R.; validation, X.L.-N., M.V.-B., D.R., and M.S.R.; formal analysis, X.L.-N., V.A.V., and M.V.-B.; investigation, X.L.-N. and M.S.R.; resources, X.L.-N.; data curation, X.L.-N.; writing—original draft preparation, X.L.-N.; writing—review and editing, X.L.-N., V.A.V., M.V.-B., D.R., and M.S.R.; supervision, V.A.V., M.V.-B., and D.R.; project administration, V.A.V.; funding acquisition, V.A.V. All authors have read and agreed to the published version of the manuscript.

Funding: This research received no external funding.

Institutional Review Board Statement: Not applicable.

Informed Consent Statement: Not applicable.

Conflicts of Interest: The authors declare no conflict of interest.

\section{References}

1. Alvarez-Campana, M.; López, G.; Vázquez, E.; Villagrá, V.A.; Berrocal, J. Smart CEI Moncloa: An IoT-Based Platform for People Flow and Environmental Monitoring on a Smart University Campus. Sensors 2017, 17, 2856. [CrossRef] [PubMed]

2. Wu, H.; Han, H.; Wang, X.; Sun, S. Research on Artificial Intelligence Enhancing Internet of Things Security: A Survey. IEEE Access 2020, 8, 153826-153848. [CrossRef]

3. Kolias, C.; Kambourakis, G.; Stavrou, A.; Voas, J. DDoS in the IoT: Mirai and Other Botnets. Computer 2017, 50, 80-84. [CrossRef]

4. Kumar, V.; Sinha, D.; Das, A.K.; Pandey, S.C.; Goswami, R.T. An Integrated Rule Based Intrusion Detection System: Analysis on UNSW-NB15 Data Set and the Real Time Online Dataset. Clust. Comput. 2020, 23, 1397-1418. [CrossRef]

5. Zarpelão, B.B.; Miani, R.S.; Kawakani, C.T.; de Alvarenga, S.C. A Survey of Intrusion Detection in Internet of Things. J. Netw. Comput. Appl. 2017, 84, 25-37. [CrossRef]

6. AL-Hawawreh, M.; Moustafa, N.; Sitnikova, E. Identification of Malicious Activities in Industrial Internet of Things Based on Deep Learning Models. J. Inf. Secur. Appl. 2018, 41, 1-11. [CrossRef]

7. Shah, A.A.; Usman, N.; Waqar, J.; Saeed, H. An Efficient Machine Learning Prediction Based Model for Intrusion Detection. In Proceedings of the 2019 International Conference on Innovative Computing (ICIC), Lahore, Pakistan, 1-2 November 2019; pp. 1-6.

8. Chaabouni, N.; Mosbah, M.; Zemmari, A.; Sauvignac, C.; Faruki, P. Network Intrusion Detection for IoT Security Based on Learning Techniques. IEEE Commun. Surv. Tutor. 2019, 21, 2671-2701. [CrossRef]

9. Larriva-Novo, X.; Vega-Barbas, M.; Villagrá, V.A.; Rivera, D.; Álvarez-Campana, M.; Berrocal, J. Efficient Distributed Preprocessing Model for Machine Learning-Based Anomaly Detection over Large-Scale Cybersecurity Datasets. Appl. Sci. 2020, 10, 3430. [CrossRef]

10. Bagui, S.; Nandi, D.; Bagui, S.; White, R.J. Classifying Phishing Email Using Machine Learning and Deep Learning. In Proceedings of the 2019 International Conference on Cyber Security and Protection of Digital Services (Cyber Security), Oxford, UK, 3-4 June 2019; pp. 1-2.

11. Kathiresan, V.; Sumathi, P. An Efficient Clustering Algorithm Based on Z-Score Ranking Method. In Proceedings of the 2012 International Conference on Computer Communication and Informatics, Coimbatore, India, 10-12 January 2012; pp. 1-4.

12. Burkov, A. The Hundred-Page Machine Learning Book; Andriy Burkov: Quebec City, QC, Canada, 2019; ISBN 1-9995795-0-X. 
13. Larriva-Novo, X.A.; Vega-Barbas, M.; Villagra, V.A.; Sanz Rodrigo, M. Evaluation of Cybersecurity Data Set Characteristics for Their Applicability to Neural Networks Algorithms Detecting Cybersecurity Anomalies. IEEE Access 2020, 8, 9005-9014. [CrossRef]

14. Ullah, I.; Mahmoud, Q.H. A Scheme for Generating a Dataset for Anomalous Activity Detection in IoT Networks. In Proceedings of the Advances in Artificial Intelligence; Goutte, C., Zhu, X., Eds.; Springer International Publishing: Cham, Switzerland, 2020; pp. 508-520.

15. Tavallaee, M.; Bagheri, E.; Lu, W.; Ghorbani, A.A. A Detailed Analysis of the KDD CUP 99 Data Set. In Proceedings of the 2009 IEEE Symposium on Computational Intelligence for Security and Defense Applications, Ottawa, ON, Canada, 8-10 July 2009; pp. 1-6.

16. Revathi, S.; Malathi, D.A. A Detailed Analysis on NSL-KDD Dataset Using Various Machine Learning Techniques for Intrusion Detectio. Int. J. Eng. Res. Technol. (IJERT) 2013, 2, 1848-1853.

17. Moustafa, N.; Slay, J. UNSW-NB15: A Comprehensive Data Set for Network Intrusion Detection Systems (UNSW-NB15 Network Data Set). In Proceedings of the 2015 Military Communications and Information Systems Conference (MilCIS), Canberra, ACT, Australia, 10-12 November 2015; pp. 1-6.

18. Maciá-Fernández, G.; Camacho, J.; Magán-Carrión, R.; García-Teodoro, P.; Theron, R. UGR ‘16: A New Dataset for the Evaluation of Cyclostationarity-Based Network IDSs. Comput. Secur. 2018, 73, 411-424. [CrossRef]

19. Mafarja, M.; Heidari, A.A.; Habib, M.; Faris, H.; Thaher, T.; Aljarah, I. Augmented Whale Feature Selection for IoT Attacks: Structure, Analysis and Applications. Future Gener. Comput. Syst. 2020, 112, 18-40. [CrossRef]

20. Modi, C.N.; Patel, D. A Novel Hybrid-Network Intrusion Detection System (H-NIDS) in Cloud Computing. In Proceedings of the 2013 IEEE Symposium on Computational Intelligence in Cyber Security (CICS), Singapore, 16-19 April 2013 ; pp. 23-30.

21. Huang, J.; Li, Y.-F.; Xie, M. An Empirical Analysis of Data Preprocessing for Machine Learning-Based Software Cost Estimation. Inf. Softw. Technol. 2015, 67, 108-127. [CrossRef]

22. Jo, J.-M. Effectiveness of Normalization Pre-Processing of Big Data to the Machine Learning Performance. J. Korea Inst. Electron. Commun. Sci. 2019, 14, 547-552. [CrossRef]

23. Özgür, A.; Erdem, H. A Review of KDD99 Dataset Usage in Intrusion Detection and Machine Learning between 2010 and 2015. PeerJ Prepr. 2016, 4, e1954v1.

24. Paulauskas, N.; Auskalnis, J. Analysis of Data Pre-Processing Influence on Intrusion Detection Using NSL-KDD Dataset. In Proceedings of the 2017 Open Conference of Electrical, Electronic and Information Sciences (eStream), Vilnius, Lithuania, 27 April 2017; pp. 1-5.

25. Salih, A.A.; Abdulrazaq, M.B. Combining Best Features Selection Using Three Classifiers in Intrusion Detection System. In Proceedings of the 2019 International Conference on Advanced Science and Engineering (ICOASE), Zakho-Duhok, Iraq, 2-4 April 2019; pp. 94-99.

26. Lokeswari, N.; Rao, B.C. Artificial Neural Network Classifier for Intrusion Detection System in Computer Network. In Proceedings of the Second International Conference on Computer and Communication Technologies; Springer: Berlin/Heidelberg, Germany, 2016; pp. 581-591.

27. Chiba, Z.; Abghour, N.; Moussaid, K.; El Omri, A.; Rida, M. A Novel Architecture Combined with Optimal Parameters for Back Propagation Neural Networks Applied to Anomaly Network Intrusion Detection. Comput. Secur. 2018, 75, 36-58. [CrossRef]

28. Hindy, H.; Brosset, D.; Bayne, E.; Seeam, A.; Tachtatzis, C.; Atkinson, R.; Bellekens, X. A Taxonomy and Survey of Intrusion Detection System Design Techniques, Network Threats and Datasets. arXiv Preprint 2018, arXiv:1806.03517.

29. Ferrag, M.A.; Maglaras, L.; Moschoyiannis, S.; Janicke, H. Deep Learning for Cyber Security Intrusion Detection: Approaches, Datasets, and Comparative Study. J. Inf. Secur. Appl. 2020, 50, 102419. [CrossRef]

30. Verma, A.; Ranga, V. Machine Learning Based Intrusion Detection Systems for IoT Applications. Wirel. Pers Commun. 2020, 111, 2287-2310. [CrossRef]

31. Ring, M.; Wunderlich, S.; Grüdl, D.; Landes, D.; Hotho, A. Flow-Based Benchmark Data Sets for Intrusion Detection. In Proceedings of the 16th European Conference on Cyber Warfare and Security, Ireland, Dublin, 29-30 June 2017; pp. 361-369.

32. Su, T.; Sun, H.; Zhu, J.; Wang, S.; Li, Y. BAT: Deep Learning Methods on Network Intrusion Detection Using NSL-KDD Dataset. IEEE Access 2020, 8, 29575-29585. [CrossRef]

33. Mulyanto, M.; Faisal, M.; Prakosa, S.W.; Leu, J.-S. Effectiveness of Focal Loss for Minority Classification in Network Intrusion Detection Systems. Symmetry 2021, 13, 4. [CrossRef]

34. Glorot, X.; Bengio, Y. Understanding the Difficulty of Training Deep Feedforward Neural Networks. In Proceedings of the Thirteenth International Conference on Artificial Intelligence and Statistics, JMLR Workshop and Conference Proceedings, Montréal, QC, Canada, 31 March 2010; pp. 249-256.

35. tf.keras.callbacks.EarlyStopping / TensorFlow Core v2.4.0. Available online: https://www.tensorflow.org/api_docs/python/tf/ keras / callbacks / EarlyStopping?hl=es-419 (accessed on 4 January 2021).

36. Cassel, M.; Lima, F. Evaluating One-Hot Encoding Finite State Machines for SEU Reliability in SRAM-Based FPGAs. In Proceedings of the 12th IEEE International On-Line Testing Symposium (IOLTS'06), Lake Como, Italy, 10-12 July 2006 ; p. 6.

37. Sklearn.Model_Selection.Train_Test_Split_Scikit-Learn 0.24.0 Documentation. Available online: https://scikit-learn.org/stable/ modules/generated/sklearn.model_selection.train_test_split.html (accessed on 3 January 2021). 
38. Glossary of Common Terms and API Elements-Scikit-Learn 0.24.0 Documentation. Available online: https://scikit-learn.org/ stable/glossary.html\#term-random_state (accessed on 3 January 2021).

39. Armbrust, M.; Das, T.; Torres, J.; Yavuz, B.; Zhu, S.; Xin, R.; Ghodsi, A.; Stoica, I.; Zaharia, M. Structured Streaming: A Declarative API for Real-Time Applications in Apache Spark. In Proceedings of the 2018 International Conference on Management of Data, Houston, TX, USA, 10-15 June 2018; pp. 601-613. 